\title{
Erythrocyte shape transformation in physiological regulation of blood viscosity
}

\author{
L. N. Katiukhin
}

Sechenov Institute of Evolutionary Physiology and Biochemistry, St. Petersburg, Russia

Email: Lion@iephb.ru

Received 29 May 2013; revised 29 June 2013; accepted 6 July 2013

Copyright (C) 2013 L. N. Katiukhin. This is an open access article distributed under the Creative Commons Attribution License, which permits unrestricted use, distribution, and reproduction in any medium, provided the original work is properly cited.

\begin{abstract}
The study was carried out to evaluate deformable and aggregative erythrocyte properties in humans under normal and pathological states and in rats exposed to various stressors. A strong correlation between the erythrocyte rheological determinants, i.e. deformability index and aggregation rate, was found under pathological and stress-induced states. The physiological significance of this phenomenon is to maintain the native structure of blood flow.
\end{abstract}

Keywords: Erythrocyte; Deformability; Aggregation; Stress

\section{INTRODUCTION}

The mammalian blood is a dilute filterable gel in which the clusters of erythrocyte aggregates can be reversibly disintegrated and formed anew in various parts of the bloodstream thereby contributing to structurization of blood flow. The blood viscosity is a variable parameter closely related to vessel geometry and blood flow rate. The changes of blood viscosity and erythrocyte shape occur continuously in organism in physiological processes of periodic alteration of $\mathrm{CO}_{2}$ concentration, the metabolites absorption and shear rate change. Therefore, the deformation and aggregation of erythrocytes as the most abundant blood cell abilities have leading importance for this process and play a crucial role in maintaining normal bloodstream structure. Probably if the bond of deformability and aggregative abilities of big mass of the red blood cells were absent, the consequences would be unpredictable.

There are few papers dealing with this interconnection between these important rheological erythrocyte parameters. Shu Chien (1970) [1] was the first to raise this problem of interrelation between deformability and aggregation. But systematic investigations of the problem have not been done. In the following years there were only occasional evidences of possible relation between them. The interdependence of erythrocyte shape, deformability and aggregation was noted as a result of their shape conversion $[2,3]$. The rheological properties of the cell have been shown to have significant effects on the dynamics of aggregation and the rate of rouleaux formation [4,5]. So, most of the authors of a few articles of recent years are inclined to believe that the deformability of red blood cells plays an important role in their aggregation capacity.

The use of suitable adequate methods for estimation of these parameters in erythrocytes under the closed-to in vivo conditions, makes it possible to elucidate substantially their relation to regulation of the viscosity blood properties by their transformation in healthy individuals, under pathology and after stress.

\section{MATERIALS AND METHODS}

All the measurements have been performed on humans and Wistar rats. The investigation deals with the whole blood of healthy volunteers (men, 20 - 24 years), breathing for $30 \mathrm{~min}$ per day via a respiratory system with initial oxygen concentration of $15.5 \%$ and $1 \%$ reducing daily. Blood samples were also obtained from patients (men, 48 - 66 years) suffering from acute coronary syndrome (ACS), stable angina of II, III and IV functional class (SA) and acute myocardial infarction (AMI) on the first admission day before drug therapy. All experiments were undertaken with the understanding and verbal consent of each subject according to the conditions set forth by a Human Subjects and Ethics Review Board. The study conforms with the Code of Ethics of the World Medical Association (Declaration of Helsinki).

Wistar rats (males, age 3 - 4 months) were subject to various stress effects: acute hypoxia in a pressure chamber for an hour corresponding to the altitude of $7600 \mathrm{~m}$ above the sea level, immobilization for 3 hours, cooling 
in a thermo-chamber at $4^{\circ} \mathrm{C}$, during complete starvation for 3 days with free water ad libitum and combination of cooling with hunger. Control rats were kept in vivarium on a standard diet. At the end of experiments the animals were decapitated using a guillotine without anesthesia. All the experiments were carried out with the approval of an Animal Protection Committee in accordance with the guidelines laid down by the NIH in the US regarding the care and use of animals for experimental procedures. Heparin in concentration of 100 units/ml was used as an anticoagulant.

The deformability of erythrocytes (ED) was studied using the method of gradient ektacytometry that allows the shape of oxygen carriers (parameter $\mathrm{O}_{\text {min }}$ ) to be assessed [6,7], using osmotic deformability profiles (osmoscans). The reversible tendency of RBC to form aggregates (REA) is described in terms of parameters determined by sequentially recording and analyzing the light transmission data through the whole heparinized blood samples at hematocrit $40 \%$ in a glass microchamber of $25 \mathrm{mcm}$ height and of $16 \mathrm{~mm}$ diameter. The full erythrocyte disaggregation shear stress is achieved by voltage increase on piezo-crystal connected with surface of the micro-chamber. The rate of spontaneous aggregation $(\mathrm{K})$ was determined from a half-period reduction of the photometric transient signal through completely disaggregated blood after power-down. All measurements were done at constant temperature of $25^{\circ} \mathrm{C}$. All results were expressed as the means \pm SD. Student's unpaired t-test was used to compare the data. Statistical significance was declared when $\mathrm{p}<0.05$.

\section{RESULTS}

The results of ED and REA obtained are represented in Table 1. Compared with healthy controls, the erythrocytes of patients with cardiovascular diseases had significantly lower EI and higher $\mathrm{K}$. The correlation between EI and $\mathrm{K}$ were calculated for each examined species. As it can be seen in Table 2, the significant direct positive correlation was revealed in the experiments: the more ED, the more EA. Figures $\mathbf{1}$ and $\mathbf{2}$ show the range

Table 1. Deformation and aggregation erythrocyte parameters of each investigated species.

\begin{tabular}{|c|c|c|c|c|c|}
\hline \multicolumn{3}{|c|}{ Men } & \multicolumn{3}{|c|}{ Rats } \\
\hline & EI, relative units & $\mathrm{K}, 10^{-3} \mathrm{~s}^{-1}$ & & EI, relative units & $\mathrm{K}, 10^{-3} \mathrm{~s}^{-1}$ \\
\hline Control, $\mathrm{n}=28$ & $0.625 \pm 0.005$ & $35.6 \pm 2.1$ & Control, $\mathrm{n}=46$ & $0.697 \pm 0.006$ & $22.9 \pm 2.9$ \\
\hline SAII, $\mathrm{n}=27$ & $0.584 \pm 0.010^{* *}$ & $47.0 \pm 0.3^{* *}$ & \multirow[b]{2}{*}{ Cooling, $n=16$} & \multirow[b]{2}{*}{$0.721 \pm 0.009^{*}$} & \multirow[b]{2}{*}{$19.8 \pm 2.3$} \\
\hline SAIII-IV, n = 41 & $0.560 \pm 0.022^{* * * *}$ & $49.1 \pm 0.4^{* * * *}$ & & & \\
\hline ACS, $n=35$ & $0.562 \pm 0.011^{\text {*** }}$ & $42.0 \pm 1.4^{*}$ & \multirow[b]{2}{*}{ Hunger, $\mathrm{n}=8$} & \multirow[b]{2}{*}{$0.706 \pm 0.012$} & \multirow[b]{2}{*}{$26.3 \pm 3.5$} \\
\hline AMI, $\mathrm{n}=19$ & $0.535 \pm 0.009^{* *}$ & $49.2 \pm 0.5^{\text {*** }}$ & & & \\
\hline Hypoxia, 7 days, $\mathrm{n}=12$ & $0.636 \pm 0.012$ & $38.1 \pm 0.8$ & Cooling + hunger, $\mathrm{n}=16$ & $0.709 \pm 0.016$ & $23.0 \pm 3.0$ \\
\hline Hypoxia, 14 days, $n=12$ & $0.653 \pm 0.009^{*}$ & $32.2 \pm 1.0$ & Immobilisation, $\mathrm{n}=16$ & $0.704 \pm 0.004$ & $39.7 \pm 4.8^{*}$ \\
\hline Hypoxia, 21 days, $\mathrm{n}=12$ & $0.645 \pm 0.007^{*}$ & $37.6 \pm 0.7$ & Hypoxia, $\mathrm{n}=16$ & $0.706 \pm 0.010$ & $43.0 \pm 5.1^{* *}$ \\
\hline
\end{tabular}

Values are means $\pm \mathrm{SD}:{ }^{*} \mathrm{p}<0.05 ;{ }^{* *} \mathrm{p}<0<0.01 ;{ }^{* * *} \mathrm{p}<0.001$.

Table 2. Correlative coefficients between EI and $\mathrm{K}$ of each investigated species.

\begin{tabular}{|c|c|c|c|}
\hline \multicolumn{2}{|c|}{ Men } & \multicolumn{2}{|c|}{ Rats } \\
\hline Control, $\mathrm{n}=28$ & $-0.010 \pm 0.025, \mathrm{p}=0.561$ & Control, $\mathrm{n}=46$ & $0.202 \pm 0.022, p=0.671$ \\
\hline SAII, $\mathrm{n}=27$ & $0.477 \pm 0.063, \mathrm{p}=0.001$ & Cooling, $\mathrm{n}=16$ & $0.592 \pm 0.028, \mathrm{p}=0.017$ \\
\hline $\mathrm{ACS}, \mathrm{n}=35$ & $0.555 \pm 0.068, \mathrm{p}=0.0004$ & Hunger, $\mathrm{n}=8$ & $0.765 \pm 0.024, p=0.027$ \\
\hline SAIII-IV, n = 41 & $0.658 \pm 0.044, \mathrm{p}=0.0008$ & Immobilisation, $\mathrm{n}=16$ & $0.768 \pm 0.013, p=0.0005$ \\
\hline AMI, $\mathrm{n}=19$ & $0.747 \pm 0.068, \mathrm{p}=0.0001$ & \multirow[b]{2}{*}{ Cooling + hunger, $\mathrm{n}=16$} & \multirow[b]{2}{*}{$0.864 \pm 0.025, p=0.006$} \\
\hline Hypoxia 7 days, $n=12$ & $0.433 \pm 0.040, \mathrm{p}=0.160$ & & \\
\hline Hypoxia 14 days, $n=12$ & $0.507 \pm 0.029, \mathrm{p}=0.093$ & \multirow[b]{2}{*}{ Hypoxia, $\mathrm{n}=16$} & \multirow[b]{2}{*}{$0.865 \pm 0.016, p=0.001$} \\
\hline Hypoxia 21 days, $\mathrm{n}=12$ & $0.695 \pm 0.018, \mathrm{p}=0.012$ & & \\
\hline
\end{tabular}




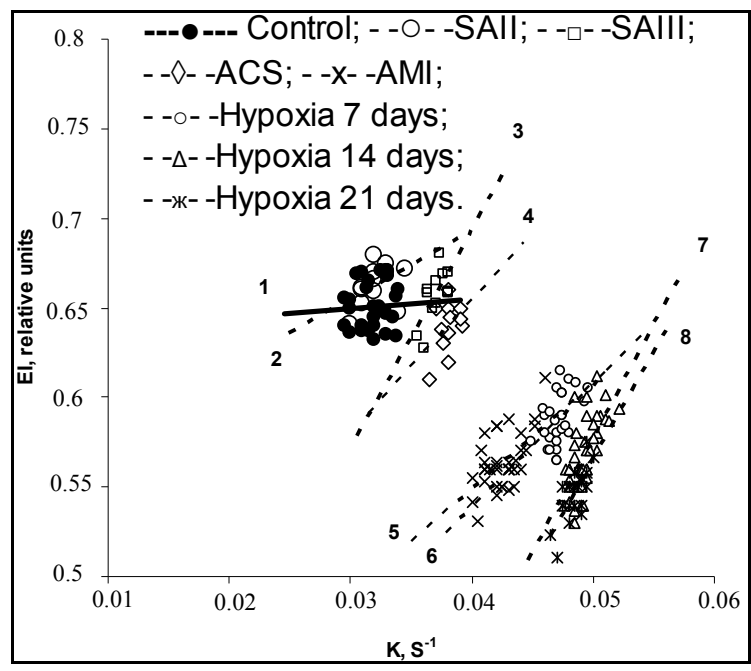

Figure 1. The strategy fields of EI versus $\mathrm{K}$ and regression lines for men.

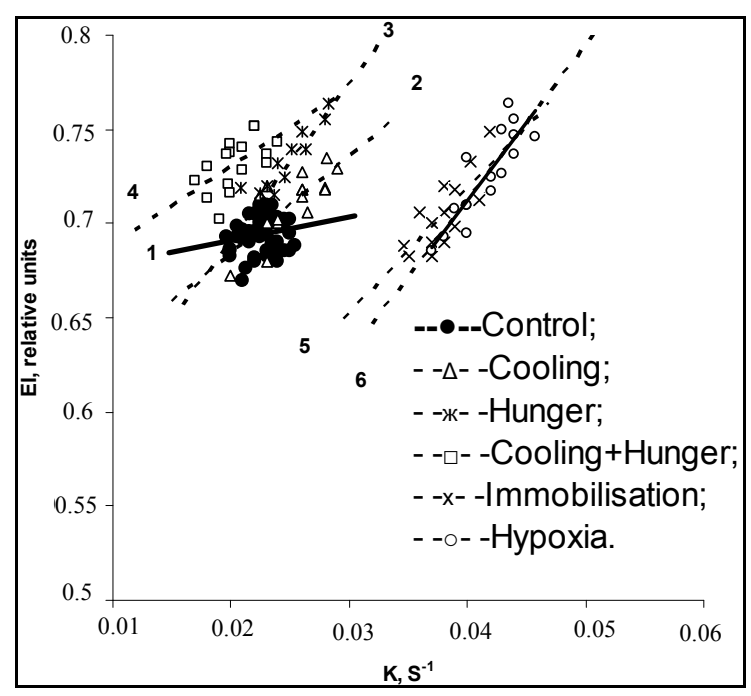

Figure 2. The strategy fields of EI versus $\mathrm{K}$ and regression lines for rats.

of EI values versus $\mathrm{K}$ and regression lines at normal and pathological states for humans and rats accordingly. Using the terminology of Population Biology it can be said that the strategy field at normal state (line 1) is described as circular distribution of the rheological determinants without any correlation between them. Another strategy field was revealed under pathological conditions (lines 2 - 8). The parameters vary in different directions in comparison with normal values, however, as follows from analysis of rheological parameter distribution, there is a close right positive correlation between them which becomes stronger in accordance with strengthening of stress effect. Thus, in all the experiments, a strong direct positive correlation dependence was revealed between ED and EA. Compared with the data obtained from healthy individuals the erythrocytes of all patients have significantly higher parameters $\mathrm{O}_{\min }$ of osmoscans, i.e. erythrocytes are spherically changed: $(147 \pm 1) \mathrm{mOsm}$ in norm and $(151 \pm 1)^{*} \mathrm{mOsm}$ in SAII, $(155 \pm 3)^{* *} \mathrm{mOsm}$ in SAIII-IV, $(180 \pm 3)^{* * *}$ mOsm in ACS, $(153 \pm 1)^{* *}$ mOsm in SAI, $(156 \pm 1)^{* *}$ mOsm in 7 days of hypoxia, $(159 \pm 2)^{* *}$ mOsm in 14 days and $(157 \pm 2)^{* *}$ mOsm in 21 days. The parameter in rats was not statistically changed. No correlation was observed between the rheological determinants and internal erythrocyte viscosity (osmoscan's parameter O').

\section{DISCUSSION}

The advantages of the osmotic gradient ektacytometry lie in its versatility, high accuracy and comprehensive characterization of red cell volume and surface maintenance. It provides information on population ED, S/V-ratio and hydration state of the erythrocyte hemoglobin. The determination of the REA-rate is more important, because the rate has dimensions to allow a dynamic physical interpretation [8]. Using whole blood samples in microchamber allows to evaluate the erythrocyte property in vivo approximation. We conducted measurements in blood samples to a single value given erythrocyte concentration of $40 \%$ since hematocrit significantly affects on the degree of the erythrocyte aggregation. This allowed us to isolate the actual changes aggregation properties of erythrocytes from other factors. Thus, the advantage of our investigation is an adequate appreciation of rheological parameters of the erythrocytes in single blood sample.

The blood viscosity results from unique abilities of red blood cells to ED and REA. It ranges essentially from $150 \mathrm{cps}$ at shear rate less than $0.1 \mathrm{~S}^{-1}$ to asymptotic values of $3.5-4 \mathrm{cps}$ while exceeding the threshold of aggregative destruction under $100-150 \mathrm{~S}^{-1}$. Such features of the blood flow curves are due to the ability of erythrocytes to form aggregates. The REA is one of the major determinants of increased blood viscosity at low shear rates. According to bridge theory, formation of monetary columns between erythrocytes is due to absorption of large molecules on the membrane surface and Van der Waals forces. Thus, the more erythrocyte profile approximates tor, the more is the area of connecting surfaces. For example, the change in erythrocyte shape (transformation) from tor to elliptic or spherical forms, which is associated with the decrease of ED due to reduction of S/V-ratio, the specific contribution of the cohesion forces decreases. The degree of REA and electrical repulsion also decrease. Since the most important thing in these transformations is the surface of interacting cells this mechanism is valid also for the theory of osmotic depletion. Under reversible erythrocyte transformations the ED enhances and amplification of electrical repulsion prevents avalanche-like strengthening of 
REA which, in our opinion, is nature occurrence in regulation of the vascular network at transformation of suspended blood elements. If there was no correlation of rheological determinants then the shape changes of abundant erythrocytes would have catastrophic for life.

One aim of practical application of the chaos theory in physiology and medicine is to establish whether the irregularity of the given process is related to the restriction of the number of linearly independent linked parameters and, correspondingly, the degree of their freedom. It was shown that the functional organization of a number of systems typical for normal state is disturbed under various diseases [9-12]. Under a lot of pathological conditions, the structural disturbances and changes in parameters of physiological regulation precede impairments in normal dynamics. Estimation of the degree of their regularity makes it possible to reveal pathological processes long before their evident manifestation. In many clinical situations a precedence of anomalous dynamics by violence of the regulation structure of physiological parameters was shown by many authors. A quantitative analysis of an order degree of them predicts future states of physiological and neurological systems [13-17]. Thus the number of connections between system parameters is diminished at pathological states, and the estimate test of organism state must be representative of their dynamic interrelation. Taking in account the conclusions of chaos theory in diagnostics of pathological states, a correlation estimate of relationship between the erythrocyte rheological determinants has been analyzed. The determinants are not related between themselves at normal state, but under pathological conditions a close direct positive relation appears between them approaching functional on some cases. Our results brought to light close ties of the rheological determinants and direct participation of erythrocyte shape in physiological regulation of blood viscosity.

The phenomenon of the direct positive connection of the rheological determinants has an important physiological significance. Under pathological violations of the cell membranes accompanied as a rule by reducing ED and strengthening REA the physiological regulation takes place. Our conclusion refreshes oneself by analyzing to their interrelations on the recovery of patients with cardiovascular diseases. According to our investigations the degree of correlative bound is reduced on the convalescence of the patients though rheological determinants are not restored. The bound accompanies a positive process and becomes near to norm, i.e. disappearance little in the time of discharging them from hospinal.

Thus, the influence of rheological determinants bound on blood viscosity is quite evident: increasing REA results in an enhanced viscosity, whereas increasing ED causes its reduction, and vice versa. Since the maintenance of blood flow structure is due to stable viscoelastic blood properties, the discovered close correlation between the rheological determinants indicates adaptive possibilities to exhibit resistance to disorders of viscous and structural homeostasis of flowing blood. The correlation coefficient between ED and EA appears to be an indicator of norm and stress in mammalian organism. The regulation counteracts an alteration of the blood viscosity by reciprocal consequences of the direct positive connection of the rheological determinants.

Thus, since supporting bloodstream structure is obliged to the blood viscosity homeostasis, the revealed phenomenon of the tight bond of rheological determinants represents an adaptive physiological mechanism in mammalian organisms to resist the break of the viscosity and structure homeostasis of moving bloodstream under pathological influences. The more the influence, the more the bond is expressed. The phenomenon has an adaptive physiological importance: under pathology of cellular membranes associated, as a rule, with impairment ED and strengthening REA, physiological regulation take place, thus preventing a shift in hemodynamic conditions and the blood viscosity due to reciprocal consequences of their direct positive relation. i.e., the enhancement of blood viscosity due to reduction of ED is compensated by REA reducing and vise versa.

\section{REFERENCES}

[1] Chien, S. (1970) Shear dependence of effective cell volume as a determinant of blood viscosity. Science, 168, 977-979. http://dx.doi.org/10.1126/science.168.3934.977

[2] Muralidharan, E., Tateishi, N. and Maeda, N. (1994) Simultaneous influence of erythrocyte deformability and macromolecules in the medium on erythrocyte aggregation: A kinetic study by a laser scattering technique. Biochimica et Biophysica Acta, 194, 255-263. http://dx.doi.org/10.1016/0005-2736(94)90307-7

[3] Reinhart, W.H. and Singh, A. (1990) Erythrocyte aggregation: The role of cell deformability and geometry. European Journal of Clinical Investigation, 20, 458-462. http://dx.doi.org/10.1111/j.1365-2362.1990.tb01884.x

[4] Bagchi, P., Johnson, P.C. and Popel, A.S. (2005) Computational fluid dynamic simulation of aggregation of deformable cells in a shear flow. Journal of Biomechanical Engineering, 127, 35-54.

http://dx.doi.org/10.1115/1.2112907

[5] Suzuki, Y., Tateishi, N., Cicha, I. and Maeda, N. (2001) Aggregation and sedimentation of mixed of erythrocytes with different properties. Clinical Hemorheology and Microcirculation, 25, 105-117.

[6] Clark, M.R., Mohandas, N. and Shohet, S.B. (1983) Osmotic gradient ektacytometry: Comprehensive characterization of red cell volume and surface maintenance. Blood, 61, 899-910.

[7] Johnson, R.M. (1989) Ektacytometry of red blood cells. Methods in Enzymology, 173, 35-54. 
http://dx.doi.org/10.1016/S0076-6879(89)73004-4

[8] Ohta, K., Gotoh, F., Tomita, M., et al. (1992) Animal species differences in erythrocyte aggregability. American Journal of Physiology-Heart and Circulatory Physiology, 262, 1009-1012.

[9] Elbert, T., Ray, W.Y., Kowalik, Z.J., et al. (1994) Chaos and physiology: Deterministic chaos in excitable cell assemblies. Physiological Reviews, 74, 1-47.

[10] Iasemidis, L.D., Sackellares, J.C., Zaveri, H.P. and Williams, W.J. (1990) Phase space topography and the Lyapunov exponent of electrocorticograms in partial seizures. Brain Topography, 2, 187-201. http://dx.doi.org/10.1007/BF01140588

[11] Kowalik, Z.J. (2000) Nonlinear mechanism of cortical oscillations. Neurologia i Neurochirurgia Polska, 34, 5365 .

[12] Lai, Y.C., Harrison, M.A., Frei, M.G. and Osorio, I. (2004) Controlled test for predictive power of lyapunov exponents: Their inability to predict epileptic seizures. Chaos, 14, 630-642. http://dx.doi.org/10.1063/1.1777831

[13] Meyer, M., Rahmel, A., Marconi, C., Grassi, B., Cerretelli, P. and Skinner, J.E. (1998) Stability of heartbeat interval distributions in chronic high altitude hypoxia. Inte- grative Physiological and Behavioral Science, 33, 344362.

[14] Mühlnickel, W., Rendtorff, N., Kowalik, Z.J., Rockstroh, B., Miltner, W. and Elbert, T. (1994) Testing the determinism of EEG and MEG. Integrative Physiological and Behavioral Science, 29, 262-269. http://dx.doi.org/10.1007/BF02691330

[15] Skinner, J.E., Carpeggiani, C., Landisman, C.R. and Fulton, K.W. (1991) Correlation dimension of heartbeat intervals is reduced in conscious pigs by myocardial ischemia. Circulation Research, 68, 966-976. http://dx.doi.org/10.1161/01.RES.68.4.966

[16] Skinner, J.E., Nester, B.A. and Dalsev, W.C. (2000) Nonlinear dynamics of heart rate variability during experimental hemorrhage in ketamine-anesthetized rats. American Journal of Physiology-Heart and Circulatory Physiology, 279, H1669-H1678.

[17] Skinner, J.E., Pratt, C.M. and Vybiral, T. (1993) Reduction in the correlation dimension of heartbeat intervals precedes imminent ventricular fibrillation in human subjects. American Heart Journal, 125, 731-743. http://dx.doi.org/10.1016/0002-8703(93)90165-6 